\title{
Evaluation of the preoperative vasoactive-inotropic score as a predictor of postoperative outcomes in patients undergoing heart transplantation
}

\author{
Eduardo Barge-Caballero, Javier Segovia-Cubero, Francisco González-Vilchez, Juan \\ Delgado-Jiménez, Félix Pérez-Villa, Luis Almenar-Bonet, José L. Arizón-Del Prado, \\ Ernesto Lage-Gallé, Luis De La Fuente-Galán, Nicolás Manito-Lorite, Marisa Sanz- \\ Julve, Adolfo Villa-Arranz, José L. Lambert Rodríguez, Vicens Brossa-Loidi, Domingo \\ Pascual-Figal, Javier Muñiz-García, Marisa Crespo-Leiro
}

Keywords

Heart transplantation; Prognosis; Vasoactive-inotropic score

\section{To the Editor:}

In patients with advanced heart failure (HF), the dependence on intravenous vasoactive drug support (VDS) is an indicator of end-stage disease associated with a lower probability of response to therapeutic interventions. It has been reported that individuals requiring vasopressors are exposed to an increased risk of right ventricular failure and death following left ventricular assist device (LVAD) implantation [1], and that preoperative VDS is a risk factor for primary graft failure after heart transplantation (HT) [2].

Clinical experience suggests that the intensity of VDS required to keep a patient stable correlates with the severity of the HF syndrome and, possibly, with clinical outcomes. This hypothesis, however, has been rarely explored, mainly due to the difficulty to compare therapeutic regimens involving a wide variety of agents and dosages. By using the vasoactive-inotropic score (VIS) - a novel clinical tool intended to express doses of different vasoactive agents in a normalized fashion that comes from the field of pediatric cardiac surgery [3] and [4] - we aimed to determine whether the intensity of preoperative VDS is a prognostic marker in patients undergoing HT.

Data for the study were collected from a multi-institutional retrospective database that contains clinical information about 711 consecutive emergency heart transplants performed in 15 Spanish hospitals between 2000 and 2009 [5] and [6]. The present analysis was restricted to 390 patients who were treated preoperatively with intravenous vasoactive drugs, and which doses were recorded. Multi-organ transplants and re-transplants were excluded. Preoperative VIS [3] and [4] was calculated as $10 \times($ dobutamine dose + dopamine dose + milrinone dose $)+100 \times$ (epinephrine dose + norepinephrine dose + vasopressin dose $)(\mu \mathrm{g} / \mathrm{kg} / \mathrm{min})$, considering drugs that patients were receiving just at the time of HT. Multivariable Cox's proportional hazards regression was used to control the effect of potential confounders - age, gender, mechanical circulatory support (MCS), mechanical ventilation, dialysis, diabetes, creatinine, prior sternotomy, cold ischaemic time, age of the donor and gender of the donor - on the association between preoperative VIS and post-transplant survival. In-hospital postoperative outcomes [5] were assessed by means of multivariable logistic regression. As in previous literature [3] and [4], high-dose preoperative vasoactive support was defined as a VIS $\geq 20$.

The study sample comprised 390 patients, of whom $74(19 \%)$ were women and $182(47 \%)$ had ischemic cardiomyopathy. Mean age of the recipients was $50 \pm 12$ years, mean age of the donors was $36 \pm 13$ years and mean waiting list time was $5.2 \pm 7$ days. Female donors were used in $108(28 \%)$ patients. Mean cold ischemic time was $215 \pm 58 \mathrm{~min}$. At the time of transplantation, $186(48 \%)$ patients were on mechanical ventilation, $32(8 \%)$ on dialysis, $273(76 \%)$ on an intraaortic balloon and $63(16 \%)$ on extracorporeal MCS. Dobutamine was used in $324(83 \%)$ patients, dopamine in $260(67 \%)$, norepinephrine in 102 (26\%), epinephrine in 33 (8\%), and milrinone in $16(4 \%)$. Mean VIS was $32 \pm 49$.

Univariable Cox's regression revealed a continuous association between higher preoperative VIS and post-transplant mortality (hazard ratio (HR) 1.005, 95\% CI 1.003-1.008, p < 0.001), which remained as statistically significant after multivariable adjustment (HR 1.005, 95\% CI 1.002-1.008, p < 0.001). 
Kaplan-Meier post-transplant survival curves in patients with a preoperative VIS $<10,10-19.9$ and $\geq 20$ are shown in Fig. 1. In-hospital postoperative outcomes are detailed in Fig. 2.

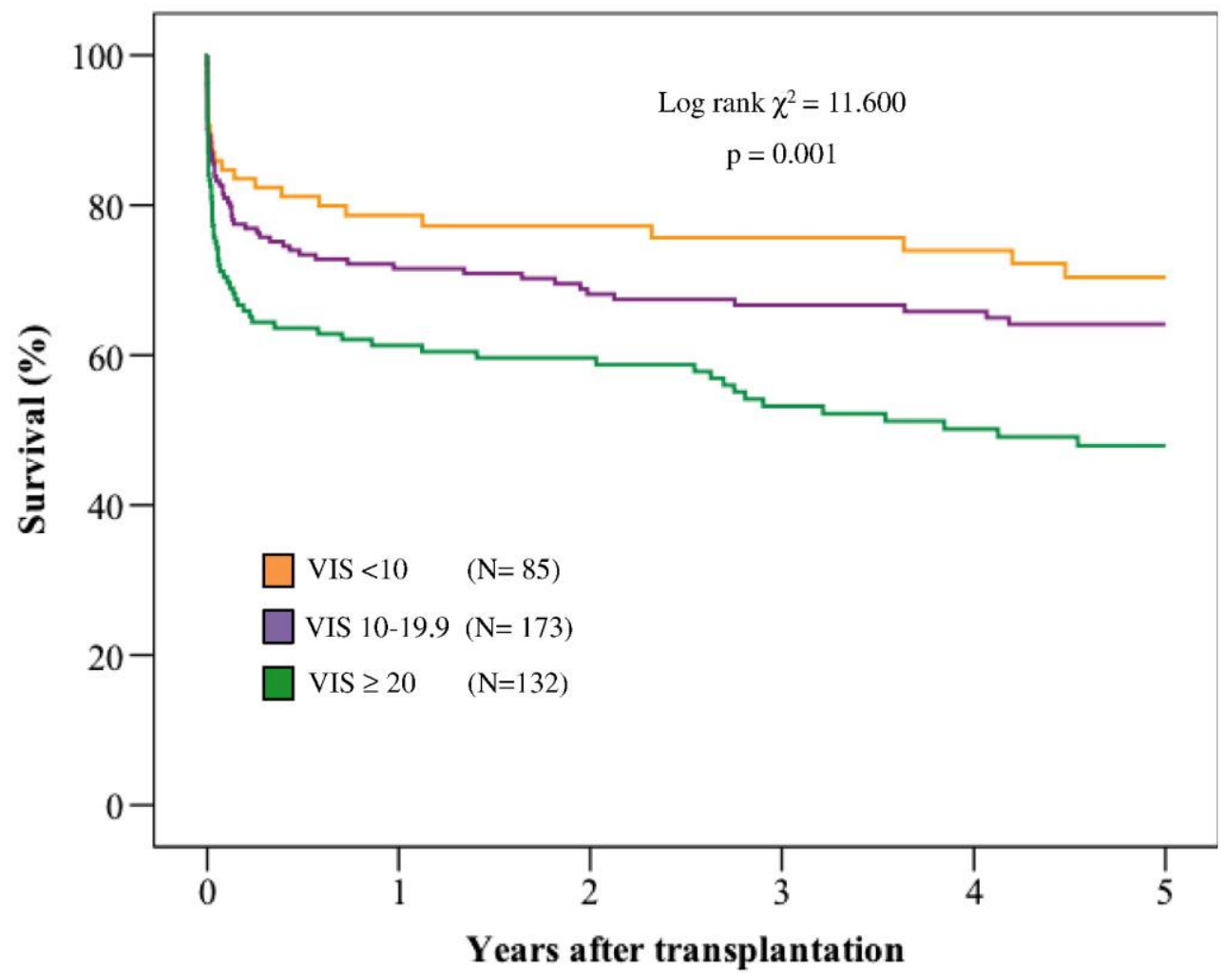

Fig. 1. Kaplan-Meier post-transplant survival curves in patients with a preoperative VIS < $10,10-19.9$ and $\geq 20$, as compared by means of the log-rank test. VIS, vasoactive-inotropic score.

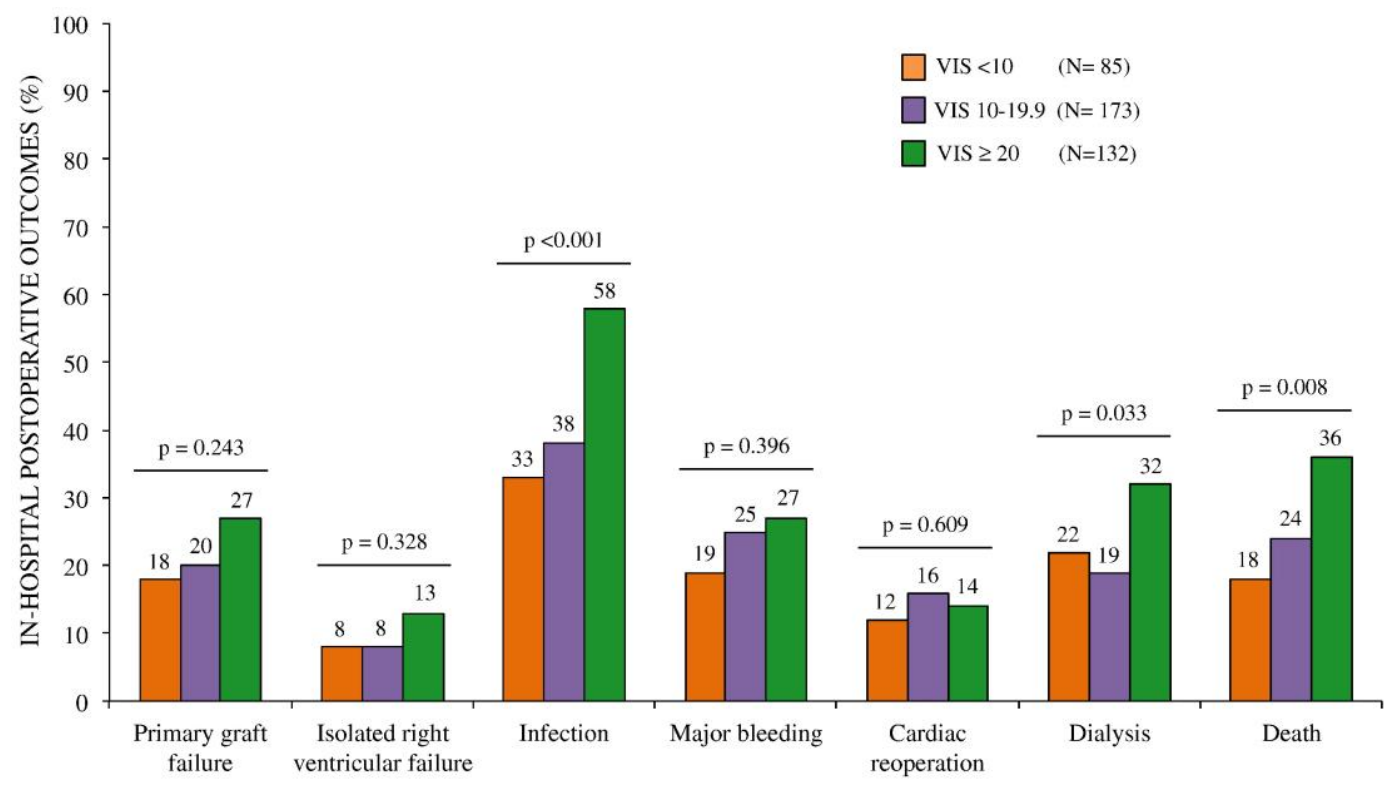

Fig. 2. In-hospital postoperative outcomes in patients with a preoperative VIS $<10,10-19.9$ and $\geq 20$. $p$ values refer to the Chisquared test. VIS, vasoactive-inotropic score. 
The need for high-dose preoperative VDS (VIS $\geq 20$ ) was identified as an independent predictor of increased post-transplant mortality, both in the entire cohort (HR 1.80, 95\% CI 1.26-2.56, $\mathrm{p}=0.001)$ and in the subcohorts of patients bridged to HT on an intraaortic balloon (HR 1.71, 95\% CI 1.09-2.69, $\mathrm{p}=0.019$ ) or on extracorporeal MCS (HR 2.66, 95\% CI 1.14-6.23, p =0.024), but not in medically managed patients (HR 0.94, 95\% CI 0.41-2.13, $\mathrm{p}=0.881$ ). By means of multivariable logistic regression, a preoperative VIS $\geq 20$ was identified as an independent predictor of postoperative infection (odds ratio (OR) $1.66,95 \%$ CI $1.02-2.71, \mathrm{p}=0.041$ ) and in-hospital postoperative mortality (OR 1.95, 95\% CI 1.17$3.28, \mathrm{p}=0.011)$.

This study confirms the hypothesis that the intensity of preoperative VDS correlates with postoperative outcomes in patients undergoing HT, and supports the routine use of the VIS for preoperative risk assessment in this population. In our multi-institutional series, we have observed that candidates requiring high-dose preoperative VDS, defined by a VIS $\geq 20$, were exposed to a significantly increased risk of post-transplant mortality, driven by a higher risk of complications and death during the early postoperative period. The adverse prognostic impact of a high preoperative VIS was especially marked among candidates bridged to transplantation under MCS. In these patients, the need for high-dose VDS may reflect an insufficient hemodynamic support - e.g., due to right ventricular failure following LVAD implantation - or other associated conditions such as sepsis, bleeding, tamponade, hypovolemia, or vasoplegia. Whatever the cause is, the resort to emergency HT as a 'bailout' therapy in this clinical scenario is associated with poor clinical outcomes and should be avoided.

\section{Conflict of interest}

None of the authors have any other conflict of interest to disclose.

\section{Acknowledgments}

Funding for this study was supplied by the Instituto de Salud Carlos III, Spanish Ministry of Economy and Competitiveness, through the Red de Investigación Cardiovascular.

\section{References}

[1].J.C. Matthews, T.M. Koelling, F.D. Pagani, et al. The right ventricular failure risk score a pre-operative tool for assessing the risk of right ventricular failure in left ventricular assist device candidates. J. Am. Coll. Cardiol., 51 (2008), pp. 2163-2172

[2].J. Segovia, M.D. Cosio, J.M. Barcelo, et al. RADIAL: a novel primary graft failure risk score in heart transplantation. J. Heart Lung Transplant., 30 (2011), pp. 644-651.

[3].M.G. Gaies, J.G. Gurney, A.H. Yen, et al. Vasoactive-inotropic score as a predictor of morbidity and mortality in infants after cardiopulmonary bypass. Pediatr. Crit. Care Med., 11 (2010), pp. 234-238

[4].M.G. Gaies, H.E. Jeffries, R.A. Niebler, S.K. Pasquali, et al. Vasoactive-inotropic score is associated with outcome after infant cardiac surgery: an analysis from the Pediatric Cardiac Critical Care Consortium and Virtual PICU System Registries. Pediatr. Crit. Care Med., 15 (2014), pp. 529-537.

[5].E. Barge-Caballero, J. Segovia-Cubero, L. Almenar-Bonet, et al. Preoperative INTERMACS profiles determine postoperative outcomes in critically ill patients undergoing emergency heart transplantation: analysis of the Spanish National Heart Transplant Registry. Circ. Heart Fail., 6 (2013), pp. 763-772.

[6].E. Barge-Caballero, L. Almenar-Bonet, A. Villa-Arranz, et al.. Impact of short-term mechanical circulatory support with extracorporeal devices on postoperative outcomes after emergency heart transplantation: data from a multi-institutional Spanish cohort. Int. J. Cardiol., 176 (2014), pp. 86-93. 